\title{
Enhanced Surveillance for Coccidioidomycosis, 14 US States, 2016
}

\author{
Kaitlin Benedict, Malia Ireland, Meghan P. Weinberg, Randon J. Gruninger, \\ Jenna Weigand, Lei Chen, Katharine Perez-Lockett, Catherine Bledsoe, Lynn Denny, \\ Katie Cibulskas, Suzanne Gibbons-Burgener, Anna Kocharian, Emilio DeBess, \\ Tracy K. Miller, Alicia Lepp, Laura Cronquist, Kimberly Warren, Jose Antonio Serrano, \\ Cody Loveland, George Turabelidze, Orion McCotter, Brendan R. Jackson
}

\begin{abstract}
Although coccidioidomycosis in Arizona and California has been well-characterized, much remains unknown about its epidemiology in states where it is not highly endemic. We conducted enhanced surveillance in 14 such states in 2016 by identifying cases according to the Council of State and Territorial Epidemiologists case definition and interviewing patients about their demographic characteristics, clinical features, and exposures. Among 186 patients, median time from seeking healthcare to diagnosis was 38 days (range $1-1,654$ days); $70 \%$ had another condition diagnosed before coccidioidomycosis testing occurred (of whom $83 \%$ were prescribed antibacterial medications); $43 \%$ were hospitalized; and $29 \%$ had culture-positive coccidioidomycosis. Most $(83 \%)$ patients from nonendemic states had traveled to a coccidioidomycosis-endemic area. Coccidioidomycosis
\end{abstract}

Author affiliations: Centers for Disease Control and Prevention, Atlanta, Georgia, USA (K. Benedict, O. McCotter, B.R. Jackson); Minnesota Department of Health, St. Paul, Minnesota, USA (M. Ireland); Michigan Department of Health and Human Services, Lansing, Michigan, USA (M.P. Weinberg); Utah Department of Health, Salt Lake City, Utah, USA (R.J. Gruninger); Southwest Utah Public Health Department, Cedar City, Utah, USA

(J. Weigand); Washoe County Health District, Reno, Nevada, USA (L. Chen); New Mexico Department of Health, Las Cruces, New Mexico, USA (K. Perez-Lockett, C. Bledsoe); Ohio Department of Health, Columbus, Ohio, USA (L. Denny, K. Cibulskas); Wisconsin Division of Public Health, Madison, Wisconsin, USA (S. Gibbons-Burgener, A. Kocharian); Public Health Division, Oregon Health Authority, Portland, Oregon, USA (E. DeBess); North Dakota Department of Health, Bismarck, North Dakota, USA (T.K. Miller, A. Lepp, L. Cronquist); Pennsylvania Department of Health, Wilkes-Barre, Pennsylvania, USA (K. Warren); Louisiana Department of Health, Baton Rouge, Louisiana, USA

(J.A. Serrano); Wyoming Department of Health, Cheyenne, Wyoming, USA (C. Loveland); Missouri Department of Health and Senior Services, St. Louis, Missouri, USA (G. Turabelidze)

DOI: https://doi.org/10.3201/eid2408.171595 can cause severe disease in residents of non-highly endemic states, a finding consistent with previous studies in Arizona, and less severe cases likely go undiagnosed or unreported. Improved coccidioidomycosis awareness in non-highly endemic areas is needed.

Cocha occidioidomycosis is a fungal infection caused by inhalation of soil-dwelling Coccidioides spp. organisms. Symptomatic infection occurs in $\approx 40 \%$ of cases and usually presents as a self-limiting, influenza-like illness (also called Valley fever) after a 1-3-week incubation period. A small proportion of patients have life-threatening severe pulmonary or disseminated disease $(1,2)$. In the United States, coccidioidomycosis is known to be endemic in the southwestern states, with hyperendemic foci in Arizona's Sonoran Desert and California's southern San Joaquin Valley (3). The disease is also endemic in parts of Nevada, New Mexico, Utah, and Texas (3) but to a lesser extent. The actual endemic areas are likely broader than previously recognized; for example, Coccidioides was found in soil in south central Washington in 2013 and was implicated in locally acquired cases (4).

Coccidioidomycosis is reportable in 22 states. To meet the Council of State and Territorial Epidemiologists (CSTE) coccidioidomycosis case definition, cases must fulfill clinical and laboratory criteria (5). Approximately 10,000 cases are reported each year through the National Notifiable Diseases Surveillance System (NNDSS), although the number varies markedly by year. NNDSS captures basic demographic information about coccidioidomycosis cases, including the patients' state and county of residence, age, sex, race, and ethnicity. Some states routinely collect additional information, such as travel history, that is not available in NNDSS. Approximately $65 \%$ of cases are reported from Arizona and $\approx 30 \%$ from California ( 6$)$, and the epidemiology and burden of coccidioidomycosis in these states has been well described. Enhanced 
surveillance in Arizona during 2007-2008 showed substantial disease, including prolonged illness, and quantified the burden on the healthcare system, including an estimated $\$ 85$ million total hospital charges in 2007 (7). In addition, patients who were aware of coccidioidomycosis before seeking healthcare were diagnosed sooner than those who did not know about the disease (7), suggesting that community awareness might prevent unnecessary diagnostic workup and antibacterial administration through earlier coccidioidomycosis diagnosis.

Although cases reported from states other than Arizona and California constitute a small proportion of total reported cases (4\% in 2015), the number generally increased during the past decade, similar to the overall trend (6), indicating that coccidioidomycosis remains a public health problem on a national scale. However, features of cases in non-highly endemic areas have not been systematically described; previous studies of non-outbreak-associated cases are limited to single-state retrospective reviews of existing surveillance data or medical chart data $(8-10)$. Therefore, we conducted enhanced surveillance in 14 states to better describe the epidemiology, diagnosis, and outcomes of these cases to help inform current routine surveillance practices and guide future awareness and educational efforts in these areas.

\section{Methods}

During January 1-December 31, 2016, routine surveillance conducted in 14 states (Louisiana, Michigan, Minnesota, Missouri, Montana, Nevada, New Mexico, North Dakota, Ohio, Oregon, Pennsylvania, Utah, Wisconsin, and Wyoming) identified coccidioidomycosis cases according to the CSTE case definition. State or local health department personnel contacted patients to participate in a voluntary telephone interview. A parent or guardian was interviewed for patients $<18$ years of age, and a relative or medical provider could complete the interview if the patient was incapacitated or deceased. Using a standardized questionnaire, health department personnel asked patients about symptoms, healthcare-seeking behaviors, diagnosis, treatment, outcomes, underlying medical conditions, and travel history. They also collected information about laboratory tests used to diagnose coccidioidomycosis from electronic surveillance databases. Some patients who met the CSTE case definition by laboratory criteria and symptoms but who clearly had a different diagnosis (such as histoplasmosis) or whose illnesses were not believed to be clinically consistent with coccidioidomycosis were not contacted for an interview. We further excluded from the analysis interviewed case-patients with compelling evidence that their illness was caused by something other than coccidioidomycosis.

We classified Nevada, New Mexico, and Utah as lowendemic because the risk for coccidioidomycosis is lower in those states than in Arizona and California. The 11 other states in this analysis were not known to be endemic for coccidioidomycosis and were considered nonendemic. We performed descriptive analysis and examined differences between cases in low- versus nonendemic states. We analyzed categorical variables by using $\chi^{2}$ or Fisher exact tests and used Wilcoxon rank-sum tests to compare continuous variables. A human subjects review by CDC determined this project to be nonresearch.

\section{Results}

\section{Interviewed and Noninterviewed Patients}

We identified 339 patients who met the CSTE coccidioidomycosis case definition. Of those, $144(43 \%)$ were not interviewed. Forty-five (31\%) of those patients were not interviewed because a different illness etiology was identified or the illness was not believed to be clinically consistent with coccidioidomycosis; another $45(31 \%)$ were unable to be contacted, $19(13 \%)$ died, $14(10 \%)$ refused, and no reason was provided for the remaining $21(15 \%)$. Among the 45 noninterviewed patients with a different illness identified or an illness not clinically consistent with coccidioidomycosis, most had histoplasmosis (18 [40\%]), 4 (9\%) had aspergillosis, and the remainder had other or unspecified diagnoses. In addition, we excluded 9 interviewed patients thought not to have coccidioidomycosis based on laboratory test results and lack of travel to endemic areas, leaving 186 interviewed patients in the final analysis. Therefore, $16 \%(54 / 339)$ of all patients did not have coccidioidomycosis, all but 1 from nonendemic states. Excluding all patients who did not have coccidioidomycosis, the response rate was $65 \%(186 / 285)$. Interviewed and noninterviewed patients were similar in age and sex.

\section{Demographic Features and Underlying Medical Conditions}

Sixty-four (34\%) patients were from low-endemic states, and 122 (66\%) were from nonendemic states; 109 (59\%) patients were male, $89 \%$ were white, and the median age was 65 (range 7-91) years (Table 1). Patients in nonendemic states were less likely than those in low-endemic states to be Hispanic or Latino ( $4 \%$ vs. $25 \%$; odds ratio [OR] 0.10, 95\% CI 0.03-0.33), were older (median 67 vs. 60 years; $p=0.01$ ), and were more likely to have a yearly household income $>\$ 50,000(61 \%$ vs. $41 \% ; p=0.043)$. The most common underlying medical conditions were diabetes (19\%), heart disease (19\%), and cancer (17\%). Patients in nonendemic states were more likely to have heart disease than those in low-endemic states (24\% vs. $9 \%$; OR $3.0,95 \%$ CI $1.2-7.7)$. Thirty-six (19\%) patients were considered to be immunosuppressed, 61 (34\%) reported no underlying conditions, and $12(7 \%)$ reported a previous history of coccidioidomycosis. 
Table 1. Demographic features and underlying medical conditions of coccidioidomycosis patients in 14 low-endemic and nonendemic US states, $2016^{*}$

\begin{tabular}{|c|c|}
\hline Characteristic & Value \\
\hline Total no. patients & $186(100)$ \\
\hline \multicolumn{2}{|l|}{ Demographics } \\
\hline \multicolumn{2}{|l|}{ Sex } \\
\hline M & 109 (59) \\
\hline $\mathrm{F}$ & $77(41)$ \\
\hline Median age, $\mathrm{y}$ (range), $\mathrm{n}=185$ & $65(7-91)$ \\
\hline \multicolumn{2}{|l|}{ Race, $n=170$} \\
\hline White & $151(89)$ \\
\hline Black/African American & $9(5)$ \\
\hline Asian & $1(0.6)$ \\
\hline American Indian or Alaska Native & $3(2)$ \\
\hline Other & $6(4)$ \\
\hline Hispanic or Latino, $\mathrm{n}=175$ & $19(11)$ \\
\hline Health insurance coverage, $n=158$ & $148(94)$ \\
\hline Some college education or higher, $n=137$ & $91(66)$ \\
\hline Annual household income $>\$ 50,000, n=104$ & $56(54)$ \\
\hline \multicolumn{2}{|l|}{ Underlying medical conditions } \\
\hline \multicolumn{2}{|l|}{ Smoking, $n=171$} \\
\hline Currently & $10(6)$ \\
\hline In the past & $74(43)$ \\
\hline None & $87(51)$ \\
\hline Asthma requiring an inhaler & $20(11)$ \\
\hline COPD or emphysema & $18(10)$ \\
\hline Other lung disease & $13(7)$ \\
\hline Diabetes & 35 (19) \\
\hline HIVIAIDS & $2(1)$ \\
\hline Heart disease & $35(19)$ \\
\hline Cancer & $32(17)$ \\
\hline Transplant & $2(1)$ \\
\hline Liver disease & $9(5)$ \\
\hline Kidney disease & $9(5)$ \\
\hline Other major illness & $49(26)$ \\
\hline No underlying medical conditions reported & $61(34)$ \\
\hline Immunosuppressed $\dagger$ & $36(19)$ \\
\hline Immunosuppressive medications, $\mathrm{n}=165$ & $32(19)$ \\
\hline History of coccidioidomycosis, $n=174$ & $12(7)$ \\
\hline
\end{tabular}

\section{Symptoms, Healthcare Use, and Diagnosis}

The most common symptoms were cough $(65 \%)$, fatigue $(62 \%)$, and shortness of breath (52\%) (Table 2). Less than half of patients reported fever $(\mathrm{n}=85$ [46\%]). Patients in nonendemic states were less likely than those in low-endemic states to report chest pain $(25 \%$ vs. $53 \%$; OR 0.30 , $95 \%$ CI $0.16-0.57)$, headache ( $24 \%$ vs. $41 \%$; OR $0.46,95 \%$ CI $0.24-0.87)$, joint pain ( $21 \%$ vs. $36 \%$; OR $0.48,95 \%$ CI $0.25-0.94)$, or muscle pain ( $18 \%$ vs. $31 \%$; OR $0.48,95 \%$ CI 0.24-0.98). Patients first sought healthcare a median of 5.5 (range 0-488; interquartile range [IQR] 1-17) days after symptom onset. Most patients first sought care at a primary care office (36\%) or emergency department (36\%). Seventy percent of patients reported receiving a diagnosis of another illness before being tested for coccidioidomycosis; among those, $63(55 \%)$ said they received a pneumonia diagnosis, and $82(83 \%)$ were prescribed antibacterial medication. Patients in nonendemic states were more likely to have had a chest radiograph performed than those in lowendemic states (94\% vs. 73\%; OR 5.4, 95\% CI 2.1-13.9).
More than half of patients (54\%) visited a healthcare provider $\geq 3$ times before being tested for coccidioidomycosis. Patients in nonendemic states were more likely than those in low-endemic states to ask for coccidioidomycosis testing $(23 \%$ vs. $10 \%$; OR $2.8,95 \%$ CI $1.1-7.2)$. Most patients were tested by a primary care physician $(30 \%)$ or a pulmonologist $(26 \%)$. Median time between seeking healthcare and diagnosis was 38 (range 1-1,654, IQR 16-73) days. Patients in nonendemic states were more likely than those in low-endemic states to have a positive coccidioidomycosis culture (36\% vs. $16 \%$; OR 3.0, 95\% CI $1.4-6.5)$ or immunodiffusion test (36\% vs. $16 \%$; OR 3.0, 95\% CI 1.4-6.5) and less likely to have a positive enzyme immunoassay test (20\% vs. $69 \%$; OR 0.12 , 95\% CI 0.06-0.23) (Table 3 ).

\section{Treatment and Outcomes}

Seventy-seven $(43 \%)$ patients were hospitalized (median duration 8 [range 3-60] days). Among 115 (68\%) patients prescribed antifungal medication, most (95 [83\%]) were prescribed fluconazole. Patients in nonendemic states were 
Table 2. Symptoms and healthcare use among coccidioidomycosis patients in 14 low-endemic and nonendemic US states, 2016*

\begin{tabular}{|c|c|}
\hline Characteristic & Value \\
\hline Symptoms & $170(91)$ \\
\hline Cough & $121(65)$ \\
\hline Fatigue & $116(62)$ \\
\hline Shortness of breath & $96(52)$ \\
\hline Fever & $85(46)$ \\
\hline Night sweats & $71(38)$ \\
\hline Chest pain & $65(35)$ \\
\hline Chills & $60(32)$ \\
\hline Weight loss & $60(32)$ \\
\hline Headache & $55(30)$ \\
\hline Rash & $54(29)$ \\
\hline Joint pain & $49(26)$ \\
\hline Muscle pain & $42(23)$ \\
\hline Wheezing & $38(20)$ \\
\hline Sore throat & $35(19)$ \\
\hline Stiff neck & $30(16)$ \\
\hline Coughing up blood & $13(7)$ \\
\hline Other symptoms & $38(20)$ \\
\hline \multicolumn{2}{|l|}{ Type of facility where patient first sought care, $n=160$} \\
\hline Emergency room & $57(36)$ \\
\hline Primary care & $57(36)$ \\
\hline Urgent care & $32(20)$ \\
\hline Specialist & $9(6)$ \\
\hline Other & $5(3)$ \\
\hline Patient first sought care in an endemic state, $n=166 \dagger$ & $105(63)$ \\
\hline Patient first sought care in Arizona, $\mathrm{n}=166$ & $46(28)$ \\
\hline Ever went to the emergency room, $n=162$ & $91(56)$ \\
\hline \multicolumn{2}{|l|}{ No. visits before being tested for coccidioidomycosis, $n=130$} \\
\hline 1 & $33(25)$ \\
\hline 2 & $27(21)$ \\
\hline$>2$ & $70(54)$ \\
\hline \multicolumn{2}{|l|}{ Type of doctor who first tested for coccidioidomycosis, $n=172$} \\
\hline Primary care & $51(30)$ \\
\hline Urgent care & $6(4)$ \\
\hline Emergency room & $16(9)$ \\
\hline Infectious disease & $30(17)$ \\
\hline Pulmonologist & $45(26)$ \\
\hline Other & $24(14)$ \\
\hline \multicolumn{2}{|l|}{ Site of infection, $\mathrm{n}=127 \ddagger$} \\
\hline Pulmonary & $105(83)$ \\
\hline Disseminated & $22(17)$ \\
\hline \multicolumn{2}{|l|}{ Total no. healthcare visits for coccidioidomycosis, $n=139$} \\
\hline 1 & $28(20)$ \\
\hline $2-3$ & $43(31)$ \\
\hline$>3$ & $68(49)$ \\
\hline Prescribed antifungal medication, $n=169$ & $115(68)$ \\
\hline Fluconazole & $95(83)$ \\
\hline Itraconazole & $13(11)$ \\
\hline Voriconazole & $4(4)$ \\
\hline Amphotericin B & $3(3)$ \\
\hline Median symptom duration, $\mathrm{d}$ (range), $\mathrm{n}=56$ & $60(7-1800)$ \\
\hline Median symptom duration among patients recovered at interview, $d$ (range), $n=44$ & $38.5(7-1800)$ \\
\hline Median symptom duration among patients not recovered at interview, $\mathrm{d}$ (range), $\mathrm{n}=12$ & $90(28-360)$ \\
\hline Median time between symptom onset and interview, $d$ (range), $n=107$ & $115(12-1672)$ \\
\hline $\begin{array}{l}\text { *Values are no. (\%) patients except as indicated. } \mathrm{n} \text { values are provided for categories with <186 resp } \\
\dagger \text { Arizona, California, Nevada, New Mexico, Texas, Utah, or Washington. } \\
\text { †Site of infection was defined as pulmonary if lungs were the only body site involved and disseminat } \\
\text { patient self-report. }\end{array}$ & ed, \\
\hline
\end{tabular}

more likely than those in low-endemic states to be prescribed antifungals (74\% vs. 57\%; OR 2.1, 95\% CI 1.09-4.1). Fiftyfour percent of patients were still symptomatic at the time of the interview; the most common symptom that these patients were still experiencing was fatigue (55\%). Among patients who had recovered at the time of the interview, median symptom duration was 38.5 (range 7-1,800, IQR 21-90) days. Coccidioidomycosis interfered with $71 \%$ of patients' usual daily activities (median number of days affected 40 [range 2-1,080] days). Among 55 (31\%) patients who had a job or were in school, 77\% missed work or school (median 19 [range 1-240] days). Four (2\%) patients died. 
Table 3. Positive laboratory tests for coccidioidomycosis among patients in 14 low-endemic and nonendemic US states, $2016^{*}$

\begin{tabular}{lc}
\hline Characteristic & Value \\
\hline Enzyme immunoassay IgM & $52(28)$ \\
Enzyme immunoassay IgM only & $20(11)$ \\
Enzyme immunoassay IgG & $40(22)$ \\
Enzyme immunoassay IgG only & $13(7)$ \\
Enzyme immunoassay lgM or IgG & $69(37)$ \\
Enzyme immunoassay lgM or IgG only & $45(24)$ \\
Immunodiffusion & $53(29)$ \\
Immunodiffusion only & $18(10)$ \\
\hline Complement fixation & $64(35)$ \\
$\quad$ Median highest complement fixation titer, $\mathrm{n}=55$ & $8(2-1024)$ \\
\hline Complement fixation only & $23(12)$ \\
Complement fixation titer 1:2 only & $7(4)$ \\
Histopathology & $9(5)$ \\
Histopathology only & $1(0.5)$ \\
\hline Culture & $53(29)$ \\
$\quad$ Bronchoalveolar lavage & $16(30)$ \\
$\quad$ Lung tissue & $16(30)$ \\
$\quad$ Sputum & $3(6)$ \\
$\quad$ Other body site & $12(23)$ \\
$\quad$ Unknown body site & $6(11)$ \\
\hline Culture only & $26(14)$ \\
Molecular evidence & $14(8)$ \\
Culture, histopathology, or molecular evidence & $61(33)$ \\
\hline${ }^{*}$ Values are no. (\%) patients except as indicated. $n$ values are provided for categories with <186 responses. & \\
\hline
\end{tabular}

\section{Travel to Known Endemic Areas}

Overall, 124 (68\%) patients (26 [41\%] from low-endemic states and 98 [83\%] from nonendemic states) traveled to Arizona, California, Nevada, New Mexico, Texas, Utah, Washington, Mexico, or Central or South America in the 4 months before symptom onset (or before testing positive, if asymptomatic) (Figures 1,2). Among 88 patients for whom information was available, median travel duration was 74.5 (range 1-720) days. Five patients, all of whom traveled to Arizona, reported that a travel partner also had coccidioidomycosis. Among patients from nonendemic states, 59 (48\%) reported part-time residence in Arizona. Of the 24 patients from nonendemic states who did not travel to known endemic areas in the 4 months before developing coccidioidomycosis, 16 reported ever traveling to those areas in their lifetime; 7 had incomplete travel histories because someone other than the patient was interviewed (n $=5$ ) or because the patient did not complete the interview $(\mathrm{n}=2)$. The remaining patient was a north-central Oregon resident with no underlying medical conditions whose only potentially relevant travel was to Mexico $\approx 12$ years before symptom onset.

\section{Knowledge of Coccidioidomycosis}

Patients in nonendemic states were more likely than those in low-endemic states to know about their positive coccidioidomycosis test results before the interview ( $90 \%$ vs. $59 \%$; OR $6.1,95 \%$ CI $2.8-13.4$ ) and were more likely to have heard of coccidioidomycosis before their diagnosis $(57 \%$ vs. $40 \%$; OR 2.0, 95\% CI 1.04-3.7). Among patients who knew about coccidioidomycosis before diagnosis, $73 \%$ first heard about it from a family member, friend, or co-worker.

\section{Discussion}

These enhanced surveillance data provide much-needed insight into coccidioidomycosis-related illness among patients with cases reported from states where the disease is not highly endemic. Our findings are generally consistent with the similar surveillance conducted in Arizona during 2007-2008 in terms of effects on patients (7). Delays in diagnosis, unnecessary antibacterial use, and prolonged symptoms were common, and a high proportion of patients had culture-positive coccidioidomycosis, suggesting that less severe cases might go undiagnosed. We found several differences between patients in low- and nonendemic states, which appear to be related to the underlying populations and testing patterns.

Patients experienced considerable diagnostic delays. Although the median time from symptom onset to seeking healthcare (5.5 days) was shorter than in Arizona surveillance (11 days), the median time from seeking healthcare to diagnosis (38 days) was longer than in Arizona (23 days) and in a study of patients in Missouri (25 days from onset to diagnosis) (7,10). Compared with Arizona surveillance, the shorter time from onset to seeking care in this investigation could be related to an older patient population, whereas the longer time from seeking healthcare to diagnosis is likely related to lower coccidioidomycosis awareness in low-endemic and nonendemic states. Many patients also reported initial misdiagnosis before being tested for coccidioidomycosis; however, it is unclear whether those who said they were first diagnosed with pneumonia did, in fact, receive a correct initial coccidioidomycosis diagnosis but were either not informed of the specific etiology or did not remember being told, or whether they were truly misdiagnosed 


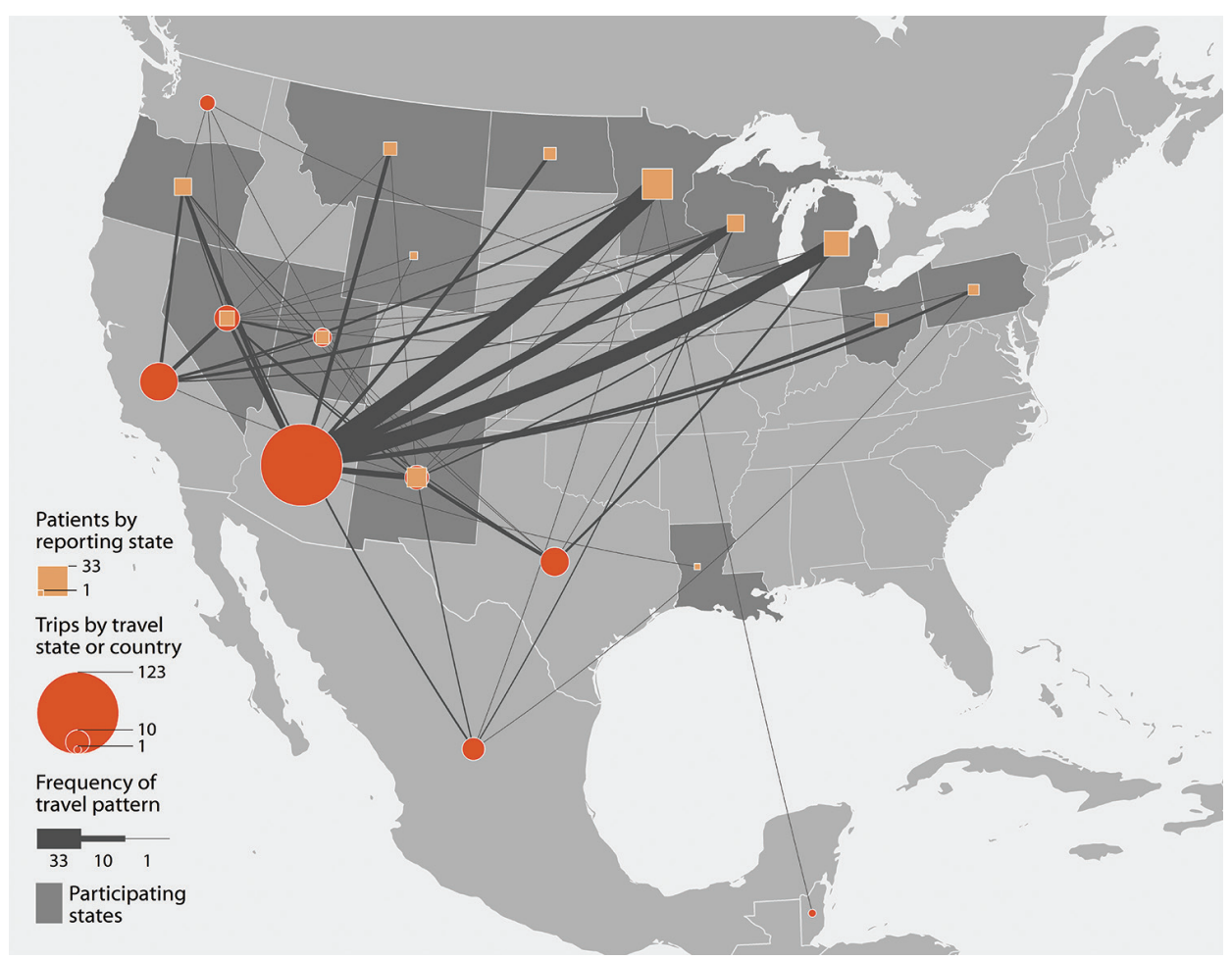

Figure 1. Reporting state and frequency of travel to coccidioidomycosis-endemic areas (Arizona, California, Nevada, New Mexico, Texas, Utah, Washington, Mexico, and Central or South America) in the 4 months before symptom onset or first positive coccidioidomycosis test among coccidioidomycosis patients reported from 14 lowendemic and nonendemic US states, 2016.

with bacterial pneumonia. Misdiagnosis seems likely because of the high proportion of patients who reported being diagnosed with another illness and receiving antibacterial medication, similar to other studies $(7,11)$. We did not observe statistically significant differences in misdiagnosis or delays in diagnosis between low-endemic and nonendemic states, indicating a need for increased healthcare provider awareness about coccidioidomycosis in all areas.

The differences between patients from low-endemic and nonendemic states appear to reflect underlying population demographics and provider testing practices rather than differences in disease severity. Enzyme immunoassay is commonly used in highly endemic areas as an initial test for coccidioidomycosis because it is high-throughput, requires less expertise, and is more sensitive (though less specific) than other serologic methods (2). Providers and laboratories in low-endemic areas might be more familiar with this test than those in nonendemic areas, whereas patients in nonendemic areas were more likely to have positive coccidioidomycosis cultures. Providers in nonendemic areas might not be testing specifically for coccidioidomycosis, but rather diagnosing it incidentally, given that Coccidioides organisms can grow on various culture media. Nearly all cultures came from invasive procedures and $<10 \%$ from sputum, suggesting that diagnosis by culture was uncommon among patients with uncomplicated primary pulmonary disease not warranting invasive procedures. Some patients from nonendemic states first sought care in endemic areas and might have had coccidioidomycosis diagnosed there; however, we did not collect data on diagnosis location, making geographic differences in testing patterns difficult to fully understand. In contrast to possible differences in providers' knowledge of coccidioidomycosis tests, patients themselves were more likely to have known about coccidioidomycosis before being diagnosed with it and were more likely to ask for coccidioidomycosis testing if they were reported from nonendemic states. The modest awareness among these patients is probably related to the fact that a high proportion resided part-time in Arizona, where public outreach about coccidioidomycosis is frequent and awareness is likely to be greater than in other areas. Patients in nonendemic states were also more likely to know about their positive results before the interview. Possible reasons that patients did not know of their positive test results include that the patient misunderstood or did not remember their diagnosis or that the provider did not inform the patient because they believed the results were not clinically relevant. The second explanation would also support the finding that patients in low-endemic states were less likely to be prescribed antifungal medications.

The most common symptoms (cough, fatigue, and shortness of breath) and prolonged symptom duration (median 38.5 days among patients who had recovered at the time of the interview) were similar to those in Arizona patients (42 days) (7). Comparable to our findings, other studies show that coccidioidomycosis symptoms, particularly fatigue, can take months to resolve and profoundly impair physical activities, resulting in missed workdays and 


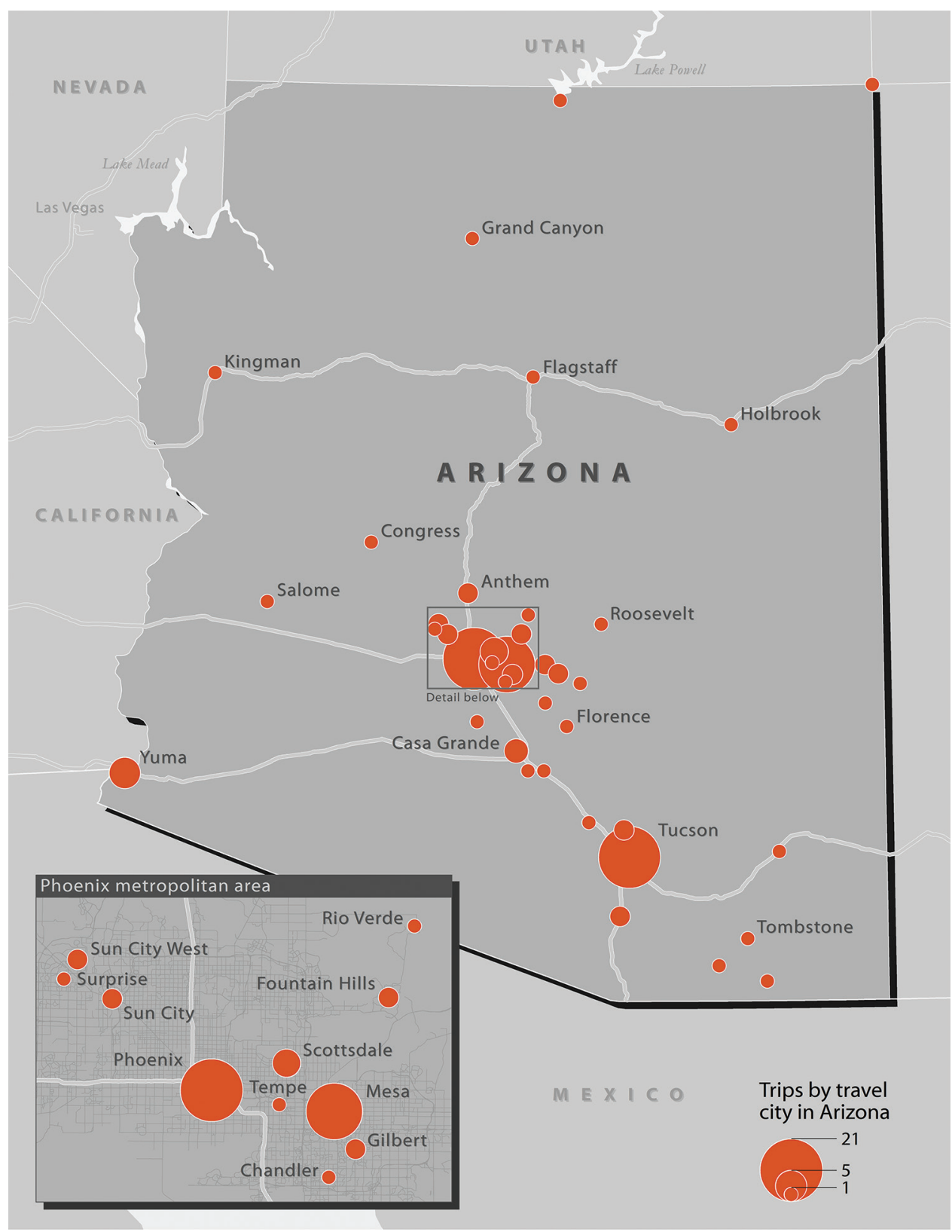

Figure 2. Frequency of trips to Arizona in the 4 months before symptom onset or first positive coccidioidomycosis test among coccidioidomycosis patients reported from 14 lowendemic and nonendemic US states, 2016.

inability to perform usual daily activities $(7,12,13)$. The reasons that patients in low-endemic states were more likely to report chest pain, headache, joint pain, and muscle pain than patients in nonendemic states are unclear but could further reflect geographic differences in testing practices if physicians in low-endemic areas are more likely to suspect and test for coccidioidomycosis based on those symptoms. In previous studies of coccidioidomycosis test positivity among community-acquired pneumonia (CAP) patients in highly endemic areas, myalgia (11) and rash (14) were the only clinical features that differentiated coccidioidal CAP from noncoccidioidal CAP. Another study found that chest pain was a significant predictor of being tested for coccidioidomycosis among CAP patients (15). In addition, approximately half of patients in our surveillance reported fever, similar to findings in Arizona surveillance (7).

Potential recall bias is our investigation's main limitation. However, patient interviews can yield insightful data about effects on patients' daily activities and other information that might not be routinely available from medical records, such as detailed travel histories.

Travel to or part-time residence in Arizona was frequent among patients reported from nonendemic states. Approximately 40 million persons visited Arizona in 2015 (16), but the number of seasonal residents is more challenging to measure; the most recent figures estimated 
Arizona's winter-only resident population to be $\approx 300,000$ during 2000-2001 (17). These seasonal residents, also known as snowbirds, are typically retired, older adults who have higher socioeconomic statuses than others in their age group and who usually cite a more enjoyable climate as the reason for their part-time residence outside their home state (18). Overall, the risk for acquiring coccidioidomycosis during travel to Arizona is likely small. One expert estimated that only 1 in 17,000 visitors would experience an infection serious enough to seek medical care (19). However, the total number of cases estimated to occur in Arizona visitors is estimated to be $\approx 1,300$ per year (19), suggesting a public health problem far larger than surveillance detects. The high proportion of cases from nonendemic states whose coccidioidomycosis was diagnosed by culture $(36 \%$, compared with $<10 \%$ in Arizona surveillance) also indicates that less severe cases go undiagnosed or unreported. Obtaining a patient's history of travel to or residence in coccidioidomycosis-endemic areas is essential for early diagnosis (2).

Some patients (59\% from low-endemic states and 17\% from nonendemic states) did not report travel to endemic areas in the 4 months before symptom onset. A study of cases in Missouri residents also found that approximately one quarter of patients did not report travel to endemic areas during the 3 weeks before symptom onset (10). Incomplete travel histories or travel that occurred $>4$ months before symptom onset likely explain the lack of recent travel to endemic areas among patients from nonendemic states in our surveillance. In low-endemic states, most cases in patients without recent travel to other endemic areas could presumably be locally acquired. A deeper understanding of the highest-risk geographic areas in those states is needed.

We classified Oregon as nonendemic; although $\mathrm{Coc}$ cidioides spp. DNA was identified from several soil samples in central Oregon in 2016, the fungus has not been cultured from environmental samples (20). Our surveillance identified culture-confirmed coccidioidomycosis in 1 Oregon patient who did not recently travel to known endemic areas, and the acute nature of his illness did not suggest reactivation of infection acquired during his earlier travel to Mexico. The patient reported extensive exposure to alfalfa hay (of unknown source), and rare coccidioidomycosis cases have been transmitted by similar fomites (21), suggesting that acquisition from the local environment and remote travel are not the only possible sources of infection. Unfortunately, a suitable clinical isolate was not available for whole-genome sequencing and comparison to isolates from nearby states. Such testing, in combination with environmental isolates, has enabled identification of cases acquired from the natural environment in south-central Washington $(4,22)$ and could allow for discovery of similar transmission in Oregon, if present.
Our results could be used to inform minor revisions to the CSTE case definition. In nonendemic areas or areas with unknown endemicity, interpretation of positive coccidioidomycosis serologic test results can be challenging if the patient is asymptomatic, has no relevant travel or an unknown travel history, or has laboratory evidence of a different disease. In this surveillance, $\geq 16 \%$ (54/339) of patients whose illness met the CSTE definition likely did not have coccidioidomycosis; all but 1 were from nonendemic states, and many had histoplasmosis, which is known to cause cross-reactions with coccidioidomycosis serologic tests (23). Some states are already excluding such cases from their case-counts even though the CSTE definition does not specify exclusion criteria. Similarly, the CSTE definition does not state whether cases counted in a previous year should be counted again if subsequent positive laboratory tests are reported. Most states, including Arizona, only count cases once because infection is thought to confer lifelong immunity. Seven percent of patients in our analysis self-reported a history of coccidioidomycosis, but we were not able to determine if their cases had been previously reported in other states. Last, $9 \%$ of patients we interviewed did not report symptoms, although the actual proportion could be higher because some patients were not contacted for an interview because their illnesses were thought to be clinically incompatible with coccidioidomycosis. In Arizona's enhanced surveillance, $5 \%$ of patients had no symptoms or symptoms inconsistent with coccidioidomycosis according to the CSTE definition, suggesting that the CSTE definition's laboratory component alone is sufficiently specific for public health surveillance (7). Further characterization of clinical scenarios involving asymptomatic patients with positive coccidioidomycosis tests could inform clinical practice and disease surveillance. Overall, the contributions of false-positive laboratory tests, previously reported cases, and asymptomatic cases to national-level case-counts are undoubtedly small, but they serve as examples of ways that coccidioidomycosis surveillance could be improved.

Although Coccidioides is most common in Arizona and California, coccidioidomycosis is a disease of national importance. Our investigation revealed many cases associated with travel to or part-time residence in highly endemic areas, as well as cases presumably acquired in Nevada, New Mexico, and Utah. Patients experienced substantial delays in diagnosis and prolonged symptoms, leading to lost productivity. The high proportion of culture-positive cases suggests that less severe cases go undiagnosed, resulting in underestimates of the actual number of cases, which is typical for public health surveillance. Greater awareness nationwide among clinicians and the public about coccidioidomycosis is needed to minimize delays in diagnosis and appropriate treatment. 


\section{Acknowledgments}

We thank Rajal K. Mody and Sekai Chideya-Chihota for contributions to the project design and William M. Hartnett for creating the figures. We also thank Zach Baucom, Jeff Brasel, Jim Collins, Carol Conroy, Michelle Feist, Louise Saw, Emily Smith, Maximilian Wegener, and state and local public health investigators who conducted patient interviews.

\section{About the Author}

Ms. Benedict is an epidemiologist in the Mycotic Diseases Branch, Division of Foodborne, Waterborne, and Environmental Diseases, National Center for Emerging and Zoonotic Infectious Diseases, CDC. Her research interests include the epidemiology and prevention of fungal infections.

\section{References}

1. Smith CE, Beard RR. Varieties of coccidioidal infection in relation to the epidemiology and control of the diseases. Am J Public Health Nations Health. 1946;36:1394-402. http://dx.doi.org/10.2105/ AJPH.36.12.1394

2. Galgiani JN, Ampel NM, Blair JE, Catanzaro A, Geertsma F, Hoover SE, et al. Infectious Diseases Society of America (IDSA) clinical practice guideline for the treatment of coccidioidomycosis. Clin Infect Dis. 2016;63:717-22. http://dx.doi.org/10.1093/cid/ ciw538

3. Edwards PQ, Palmer CE. Prevalence of sensitivity to coccidioidin, with special reference to specific and nonspecific reactions to coccidioidin and to histoplasmin. Dis Chest. 1957;31:35-60. http://dx.doi.org/10.1378/chest.31.1.35

4. Marsden-Haug N, Hill H, Litvintseva AP, Engelthaler DM, Driebe EM, Roe CC, et al. Coccidioides immitis identified in soil outside of its known range-Washington, 2013. MMWR Morb Mortal Wkly Rep. 2014;63:450.

5. Council of State and Territorial Epidemiologists. Coccidioidomycosis/ Valley fever (Coccidioides spp.) 2011 case definition [cited $2017 \mathrm{Apr}$ 12]. https://wwwn.cdc.gov/nndss/conditions/coccidioidomycosis/ case-definition/2011

6. CDC. Valley fever (coccidioidomycosis) statistics [cited 2018 Mar 19]. https://www.cdc.gov/fungal/diseases/coccidioidomycosis/ statistics.html

7. Tsang CA, Anderson SM, Imholte SB, Erhart LM, Chen S, Park BJ, et al. Enhanced surveillance of coccidioidomycosis, Arizona, USA, 2007-2008. Emerg Infect Dis. 2010;16:1738-44. http://dx.doi.org/10.3201/eid1611.100475

8. Chaturvedi V, Ramani R, Gromadzki S, Rodeghier B, Chang HG, Morse DL. Coccidioidomycosis in New York State. Emerg Infect Dis. 2000;6:25-9.

9. Desai SA, Minai OA, Gordon SM, O’Neil B, Wiedemann HP, Arroliga AC. Coccidioidomycosis in nonendemic areas: a case series. Respir Med. 2001;95:305-9. http://dx.doi.org/10.1053/ rmed.2000.1039
10. Turabelidze G, Aggu-Sher RK, Jahanpour E, Hinkle CJ. Coccidioidomycosis in a state where it is not known to be endemic - Missouri, 2004-2013. MMWR Morb Mortal Wkly Rep. 2015;64:636-9.

11. Valdivia L, Nix D, Wright M, Lindberg E, Fagan T, Lieberman D, et al. Coccidioidomycosis as a common cause of communityacquired pneumonia. Emerg Infect Dis. 2006;12:958-62. http://dx.doi.org/10.3201/eid1206.060028

12. Blair JE, Chang YH, Cheng MR, Vaszar LT, Vikram HR, Orenstein R, et al. Characteristics of patients with mild to moderate primary pulmonary coccidioidomycosis. Emerg Infect Dis. 2014;20:983-90. http://dx.doi.org/10.3201/eid2006.131842

13. Garrett AL, Chang YH, Ganley K, Blair JE. Uphill both ways: fatigue and quality of life in Valley fever. Med Mycol. 2016;54:310-7. http://dx.doi.org/10.1093/mmy/myv097

14. Kim MM, Blair JE, Carey EJ, Wu Q, Smilack JD. Coccidioidal pneumonia, Phoenix, Arizona, USA, 2000-2004. Emerg Infect Dis. 2009;15:397-401. http://dx.doi.org/10.3201/ eid1563.081007

15. Chang DC, Anderson S, Wannemuehler K, Engelthaler DM, Erhart L, Sunenshine RH, et al. Testing for coccidioidomycosis among patients with community-acquired pneumonia. Emerg Infect Dis. 2008;14:1053-9. http://dx.doi.org/10.3201/eid1407.070832

16. Arizona Office of Tourism. Economic impact of the travel industry in Arizona [cited 2017 Apr 18]. https://tourism.az.gov/ research-statistics/economic-impact

17. Hogan T, Happel S. Survey finds little change in winter resident population. Arizona Business. 2001;28:5-7.

18. Franklin A, Raadschelders J. Tracking invisible residents: how does this phenomenon impact city government? Journal of Public Budgeting, Accounting, and Financial Management. 2007;19: 488-513. http://dx.doi.org/10.1108/JPBAFM-19-04-2007-B005

19. Galgiani JN. Cocci in Arizona visitors: What are the chances? Sombrero Magazine. Tucson, AZ: Pima County Medical Society; 2011. p. 28-9.

20. Hawryluk M. Traces of Valley fever fungus found in central Oregon. The Bulletin [cited 2016 Jul 28]. http://www.bendbulletin. com/home/4524652-151/traces-of-valley-fever-fungus-foundin-central

21. Albert BL, Sellers TF Jr. Coccidioidomycosis from fomites. Report of a case and review of the literature. Arch Intern Med. 1963; 112:253-61. http://dx.doi.org/10.1001/archinte.1963. 03860020151021

22. Litvintseva AP, Marsden-Haug N, Hurst S, Hill H, Gade L, Driebe EM, et al. Valley fever: finding new places for an old disease: Coccidioides immitis found in Washington State soil associated with recent human infection. Clin Infect Dis. 2015;60:e1-3. http://dx.doi.org/10.1093/cid/ciu681

23. Wheat J, French ML, Kamel S, Tewari RP. Evaluation of cross-reactions in Histoplasma capsulatum serologic tests. J Clin Microbiol. 1986;23:493-9.

Address for correspondence: Kaitlin Benedict, Centers for Disease Control and Prevention, 1600 Clifton Rd NE, Mailstop C09, Atlanta, GA 30329-4027, USA; email: jsy8@cdc.gov 